\title{
Looking for building blocks of the Galactic halo: variable stars in the Fornax, Bootes I, Canes Venatici II dwarfs and in NGC 2419
}

\author{
Claudia Greco, ${ }^{1}$ Gisella Clementini, ${ }^{1}$ E. V. Held,${ }^{2}$ E. Poretti, ${ }^{3}$ \\ M. Catelan, ${ }^{4}$ L. Federici, ${ }^{1}$ M. Maio, ${ }^{1}$ M. Gullieuszik, ${ }^{2}$ V. Ripepi,${ }^{5}$ \\ M. Dall'Ora ${ }^{5}$ L. Di Fabrizio,${ }^{6}$ K. Kinemuchi,,${ }^{7,8}$ M. Di Crescienzo,${ }^{9}$ \\ M. Marconi, ${ }^{5}$ I. Musella, ${ }^{5}$ B. Pritzl,${ }^{10}$ A. Rest,${ }^{11}$ N. De Lee ${ }^{8}$ and \\ H. Smith ${ }^{12}$
}

${ }^{1}$ INAF - Bologna, Italy, ${ }^{2}$ INAF - Padova, Italy ${ }^{3}$ INAF - Brera, Italy, ${ }^{4}$ Pontifícia Universidad Católica, Chile, ${ }^{5} \mathrm{INAF}$ - Naples, Italy ${ }^{6} \mathrm{INAF}$ - La Palma, Italy ${ }^{7}$ Universidad de Concepción, Chile, ${ }^{8}$ University of Florida, USA, ${ }^{9}$ INAF - Rome, Italy, ${ }^{10}$ University of Wisconsin, Oshkosh, USA, ${ }^{11}$ Harvard-Smithsonian Center for Astrophysics, USA, ${ }^{12}$ Michigan State University, USA

\begin{abstract}
Lambda$ cold-dark-matter hierarchical models of galaxy formation suggest that the halo of the Milky Way (MW) has been assembled, at least in part, through accretion of protogalactic fragments partially resembling the present-day dwarf spheroidal (dSph) satellites of the MW. Investigation of the stellar populations of the MW's globular clusters (GCs) and dSph companions can thus provide excellent tests to infer the dominant Galaxy-formation scenario, whether merger/accretion or cloud collapse. Pulsating variable stars offer a very powerful tool in this context, since variables of different types allow tracing the different stellar generations in a galaxy and to reconstruct the galaxy's star-formation history and assembly back to the first epochs of galaxy formation. In particular, the RR Lyrae stars, belonging to the old population $(t>10 \mathrm{Gyr})$, witnessed the epoch of halo formation, and thus hold a crucial role to identify the MW satellites that may have contributed to build up the Galactic halo. In the MW, most GCs with an RR Lyrae population sharply divide into two distinct groups (Oosterhoff types I and II) based on the mean periods and relative proportion of fundamental-mode (RRab) and first-overtone (RRc) RR Lyrae stars. On the other hand, the Galactic-halo field RR Lyrae stars show a dominance of Oosterhoff I properties. Here, we investigate the Oosterhoff properties of a number of different stellar systems, starting from relatively undisturbed dwarf galaxies (the Fornax dSph and its globular clusters), through distorted and tidally disrupting ones (the Bootes and Canes Venatici II dSphs), to possible final relics of the disruption process (the Galactic globular cluster NGC 2419). We are addressing the crucial question of whether the RR Lyrae pulsation properties in these systems conform to the Oosterhoff dichotomy characterizing the MW variables. If they do not, the Galaxy's halo cannot have been assembled by dSph-like protogalactic fragments resembling the present-day dSph companions of the MW. We have reduced and combined long time series from different telescopes, both ground- and space-based. Variable stars have been detected with image-subtraction techniques using the package IsIs2.1. Periods, amplitudes and Oosterhoff type for all variable stars, as well as color-magnitude diagrams of the stellar populations are discussed for each stellar cluster analyzed.
\end{abstract}

Keywords. stars: variables: other, Galaxy: halo, galaxies: dwarf, Local Group

The full poster (in pdf format) is available at http://www. astro.iag.usp.br/ iaus266/Posters/pGreco.pdf. 